$54^{\text {ème }}$ Congrès de la SFMBCB, 03014 (2011)

DOI: $10.1051 / \mathrm{sfmbcb} / 20115403014$

(C) Owned by the authors, published by EDP Sciences, 2011

\title{
Kyste dentigère chez l'enfant : intérêt de la décompression. Présentation de deux cas cliniques
}

\author{
Chbicheb S, El Wady W \\ Service d'Odontologie chirurgicale, CCTD, Rabat, Maroc \\ s_chbicheb@yahoo.fr
}

Le kyste dentigère ou folliculaire représente la pathologie kystique la plus fréquente chez les enfants de moins de 12 ans. Il se développe au contact d'une dent dont les racines ne sont pas encore formées. Il constitue le plus souvent une complication de l'atteinte pulpaire de la dent temporaire qui s'étend au germe sous-jacent. Cette lésion ostéolytique se développe aux dépens des éléments épithéliaux du sac folliculaire par accumulation de sérosité entre la couronne déjà formée et l'épithélium adamantin réduit (Moulis 2003). L'image kystique déborde l'espace interradiculaire de la dent temporaire du germe. En l'absence de traitement, le kyste refoule le germe et s'étend d'autant plus facilement que, chez l'enfant, le tissu osseux est peu minéralisé (Decelli 2002). Il siège de façon préférentielle sur la deuxième prémolaire inférieure : en effet, les deuxièmes molaires temporaires sont fréquemment cariées et les rapports entre les racines de ces molaires et le germe sous-jacent sont très étroits (Harnet 1997).

Le traitement des kystes folliculaires est généralement chirurgical, mais leur énucléation peut provoquer la perte d'un ou plusieurs germes de dents permanentes. Parmi les méthodes conservatrices, c'est la décompression kystique. Le protocole clinique consiste à utiliser une technique de décompression, visant à éliminer le phénomène de pression hydrostatique régnant à l'intérieur de la cavité kystique, qui est responsable de la lyse osseuse, et à préserver les germes des dents permanentes en rapport avec la lésion kystique.

Un enfant, âgé de 10 ans, s'est présenté dans le service pour une tuméfaction siégeant en regard de la région prémolaire inférieure droite. L'examen clinique a montré une tuméfaction de consistance dure, dépréssible à son centre, s'étendant de la 83 jusqu'à la 46, recouverte d'une muqueuse d'aspect normal. L'examen radiologique a révélé une image radioclaire entourant la couronne du germe de la 44 et s'étendant jusqu'à la face mésiale du germe de la 45 ; cette image est également en rapport avec les racines de la 84. Devant ces renseignements cliniques et radiologiques, le diagnostic du kyste folliculaire est évoqué. La technique de décompression a consisté en l'ouverture de la cavité kystique après une incision en collorette au niveau du point le plus déclive de la muqueuse de recouvrement, réalisation d'une fenêtre osseuse, excision de la paroi du kyste en regard afin de réduire la pression dans la cavité kystique, et de confirmer la nature de la lésion par un examen anatomopathologique. Puis on a placé dans la cavité un tube de drainage en polyéthylène, fixé par des points de suture à la muqueuse vestibulaire. Un lavage quotidien de la cavité kystique a été réalisé à travers le tube avec de la chlorhéxidine à $0,1 \%$ à l'aide d'une seringue. Le patient a effectué des irrigations bi-quotidiennes et des contrôles cliniques et radiologiques réguliers. Après 11 mois, les résultats ont montré une disparition complète de la lésion kystique et la 44 a fait une éruption normale. 